\title{
Apolipoprotein B to Apolipoprotein A1 Ratio Measurement
}

National Cancer Institute

\section{Source}

National Cancer Institute. Apolipoprotein B to Apolipoprotein A1 Ratio Measurement. NCI

Thesaurus. Code C103356.

The determination of the ratio of the Apolipoprotein B compared with Apolipoprotein A1 in a sample. The measurement may be expressed as a ratio or percentage. 